\title{
Genetic Cross-Talk During Head Development in Drosophila
}

\author{
Amr Amin* \\ Biology Department, Faculty of Science, University of UAE, PO Box 17551, Al Ain, United Arab Emirates
}

Received 22 February 2003; accepted 3 June 2003

\begin{abstract}
The dorsal head vertex of Drosophila is specified mainly by the orthodenticle (otd) gene. The expression and the function of otd are regulated by the concerted action of many genes including hedgehog $(h h)$ and notch $(N)$. These genes are components of a meshwork of signaling transduction pathways that interact to form the dorsal head capsule of the fruit fly. Loss-of-function $H h$ mutants lack ocelli; however, loss-of-function $N$ mutants lack a different domain of the dorsal head vertex. This report provides new evidence that the $H h$ and $N$ pathways are two epistatic signaling cascades that act genetically upstream of the dorsal head capsule specification gene.
\end{abstract}

\section{INTRODUCTION}

A breakthrough in understanding the Drosophila head development came with the identification of the orthodenticle (otd) homeobox gene. Previous analysis indicated that the otd transcription factor acts as an essential regulatory gene for establishing the eyes, antenna, and parts of brain and for the dorsal head development $[1,2]$. The Drosophila dorsal head capsule includes the ocellar, frons, and orbital cuticles. The ocelli are three simple lightsensitive organs on the ocellar cuticle. The development of the dorsal head occurs during the larval stages from the dorsal head primordium in eye disc. The ocellar cuticle is formed by a fusion of the two eye discs, with the medial ocellus receiving contributions from both discs.

Previous work revealed several genes that might interact with otd in embryonic or imaginal disc development. Several of these are expressed in the dorsal head primordium and loss of their functions produces an otdlike phenotype. Hh signaling plays crucial roles in the development of Drosophila and vertebrate embryos. In the fly, there is a single hedgehog $(h h)$ gene. In contrast, three different genes, sonic hedgehog (Shh), Indian hedgehog $(I h h)$, and desert hedgehog (Dhh) play distinct regulatory roles in mammals [3]. As a segment polarity gene, $\mathrm{Hh}$ initiates a conserved signaling cascade in a variety of developmental processes [4]. Hh protein binds to a multipass membrane protein, Patched (Ptc), and prevents it from inhibiting the function of a second transmembrane protein, Smoothened (Smo). This allows Smo to signal through the positive actions of Fused (Fu) and Cubitus interruptus (Ci) and the inhibitory effects of Costal2 (Cos2) and Drosophila Protein kinase A (dPKA). In other words,
Ptc inhibits the activity of Smo, and consequently $\mathrm{Hh}$ binding to Ptc releases Smo from this inhibitory process $[5,6,7,8]$ enabling Smo to interact with $\mathrm{Fu}, \mathrm{Ci}, \mathrm{Cos} 2$, and dPKA.

$\mathrm{Ci}$ is a transcriptional effector of Hh signaling (reviewed in [9]). The expression of $c i$ mRNA is repressed by engrailed (En) protein in both embryos and imaginal discs [10]. $\mathrm{Ci}$ is also posttranslationally regulated. In the absence of $\mathrm{Hh}$ stimulation, $\mathrm{Ci}$ is cleaved into a smaller $\mathrm{N}$-terminal fragment (Ci75) which moves to the nucleus and represses Hh target genes. Upon secretion, Hh binds to Ptc and relieves the inhibitory effect that Ptc normally has on Smo. Once it is freed of the inhibitory effects of Ptc, Smo signals through unknown mechanisms to the $\mathrm{Fu} / \mathrm{Cos} 2 / \mathrm{Ci}$ complex causing hyperphosphorylation of $\mathrm{Fu}$ and $\operatorname{Cos} 2$ and causing the complex to loosen its hold on microtubules. The Hh signaling thus increases the level of Ci155 (Figure 1) by repressing the cleavage of Ci155 into Ci75 and/or by releasing it from a microtubule-bound ci$\mathrm{Fu}-\mathrm{Cos} 2$ complex. The full-length Ci155 can then travel to the nucleus and function as a transcriptional activator, upregulating transcription of Hh target genes [11, 12].

Both Ci75 and Ci155 contain the zinc finger domain that is capable of binding to DNA sequence in Hh target genes [13]. Ci75 acts as a repressor and represses the expression of $h h$, ptc, and wingless $(\mathrm{wg})$. It also contains a domain that can bind to the Drosophila cAMP-responseelement-binding protein (CREB)-binding protein $(\mathrm{CBP})$ protein (Figure 1).

Several components of the Hh signaling cascade show region-specific expression in the ocellar region. Both $h h$ and $p t c$, a gene that encodes a receptor for $h h[14]$, have strong expression in the vertex primordium. While the 


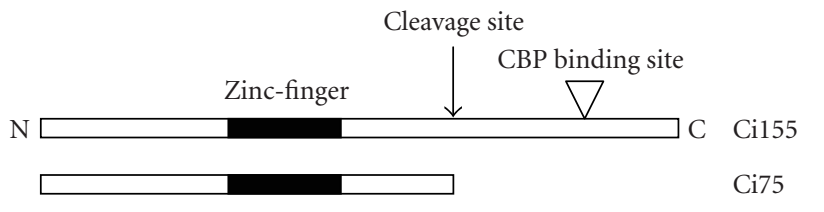

(a)

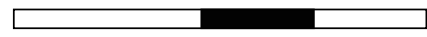

Ci76

(b)

FIgURE 1. Schematic representation of $\mathrm{Ci}$ protein isoforms and upstream activating sequence (UAS) constructs. (a) In vivo forms of $\mathrm{Ci}$. Ci155 and $\mathrm{Ci} 75$ are two forms of $\mathrm{Ci}$ that are produced during the normal course of development, $\mathrm{Ci}$ is a zinc-finger protein homologous to proteins in vertebrate GLI family. Ci75 is normally produced during development by the cleavage of the full-length Ci155 protein at the shown site. cAMP-response-element-binding protein (CREB)-binding protein (CBP)-binding site is located close to the C-terminus. (b) UAS constructs of $\mathrm{Ci}$. Ci76 is the UAS construct used in this investigation to recapitulate the function of the in vivo repressor form of $\mathrm{Ci}$ (Ci75).

role of ptc in the ocellar region is not known, $h h$ is required for the ocellar development. Loss of $h h$ function leads to a loss-of-ocelli phenotype while ectopic expression results in supernumerary and larger ocelli [15]. ci and $f u$ genes are also implicated in the process of ocellar formation [16].

Although both otd and $h h$ are involved in ocellar development, their relationship is unclear. In the vertex primordium, $h h$ is expressed within the otd-expressing domain. Consistent with this observation, $h h$ is required for ocellar cuticle development while otd specifies both the ocellar and frons cuticular structures. Additionally, ectopic $h h$ is able to turn on the expression of otd ectopically in the eye disc [17].

$\operatorname{Notch}(N)$ is another gene that is crucial for the head formation in Drosophila. It encodes a large transmembrane protein with an extracellular domain containing 36 epidermal growth factor (EGF)-like repeats and an intracellular domain including 6 ankyrin repeats [18]. Studies in Drosophila and other organisms demonstrated that the $\mathrm{N}$ protein is a receptor that regulates the differentiation of a wide range of cell types. These analyses suggest that $\mathrm{N}$ and other components of the $\mathrm{N}$ signaling pathway control the ability of uncommitted cells to respond to specific developmental signals, thereby regulating their progression to a committed state [19].

Originally, $N$ was shown to be involved in neural fate determination in the Drosophila embryo. Strong $\mathrm{N}$ mutations cause a failure of neuroblast-mediated lateral inhibition, leading to hypertrophy of the nervous system at the expense of epidermis. Similarly, the loss of $N$ function during postembryonic stages leads to supernumerary sensory organ precursors [20]. $N$ is also expressed in many other tissues throughout Drosophila development [21]. N activity is required for the proper formation of the meso- derm, germ line, wing margin, and compound eyes [22]. Homologues of the $N$ gene have been identified in other species, where they appear to function in an analogous fashion $[23,24]$.

Genetic and molecular studies in Drosophila identified a group of genes that participate in $\mathrm{N}$-mediated signaling. These include delta $(D l)$ and serrate (Ser), which encode putative extracellular ligands for $\mathrm{N}$; deltex $(d x)$, which encodes a cytoplasmic protein that binds to $\mathrm{N}$ ankyrin repeats; and hairless $(H)$ and suppressor of hairless $(S u(H))$, mastermind (mam), and the enhancer of split complex $(E(s p l)-C)$, all of which encode nuclear proteins. Dl and Ser bind to the extracellular EGF repeats, while Dx interacts with $\mathrm{N}$ in the vicinity of the ankyrin repeats. The $\mathrm{Su}(\mathrm{H})$ protein translocates to the nucleus, where it activates the transcription of target genes of the $\mathrm{N}$ pathway [25].

The $E(s p l)$ gene complex encodes a group of transcription factors that act downstream of $\mathrm{N}$ in many Drosophila tissues. Accumulation of $\mathrm{E}(\mathrm{spl})$ proteins during embryonic and imaginal development depends on $\mathrm{N}$ activation and is mediated by $\mathrm{Su}(\mathrm{H})$ function. The $E(s p l)$ region transcript involves $E(s p l) m 8, E(s p l) m 7$, $E(s p l) m 5, E(s p l) m 3, E(s p l) m \delta, E(s p l) m \gamma$, and $E(s p l) m \beta$. These genes encode seven closely related basic helix-loophelix (bHLH) protein family of transcription factors (m3, $\mathrm{m} 5, \mathrm{~m} 7, \mathrm{~m} 8, \mathrm{~m} \beta, \mathrm{m} \gamma$, and $\mathrm{m} \delta$ ) [26]. Genetic and molecular analyses suggest an overlap in the functions of these proteins. Consistent with this proposed functional redundancy, the patterns of expression of the different $E(s p l)$ bHLH genes are very similar.

This study is an investigation of the genetic interaction between $\mathrm{Hh}$ and $\mathrm{N}$ signaling pathways during the development of the head capsule of the adult fruitfly. Both $\mathrm{Hh}$ and $\mathrm{N}$ pathways are shown to be required for the formation of the medial region of the dorsal head. Loss of $\mathrm{Hh}$ signaling causes the deletion of a specific dorsal head region where $\mathrm{N}$ is normally active.

\section{MATERIALS AND METHODS}

\section{Stocks}

The wild-type strain used was Oregon-R. $h h^{t s 2}$ and $N^{t s 1}$ are temperature-sensitive alleles that fail to produce functional $\mathrm{Hh}$ and $\mathrm{N}$ proteins, respectively, at $29^{\circ} \mathrm{C}$ $[27,28]$. The Gal4 strain used is Gal4 $4^{C 591}$ in which Gal4 driver activates gene expression across the entire dorsal head primordium [29]. The upstream activating sequence (UAS)-ptc is a gift from E Wilder. UAS-E(spl)m8, UAS$E(s p l) m 7$, and $U A S-E(s p l) m \beta$ were a gift from JF de Celis. Reporter genes used are $p t c-l a c Z$ and $p\{17.6(\mathrm{~L} 1)-\mathrm{lac} Z$ \} line (L1) [30]. The lacZ enhancer trap L1 labels ocelli precursor cells and cells in the lamina of the brain. Stocks were maintained on cornmeal-yeast-agar medium seeded with baker's yeast at $25^{\circ} \mathrm{C}$. For temperature shift experiments, flies were transferred to $29^{\circ} \mathrm{C}$ at late larval stages for 12-15 hours. 


\section{Fly crossing to study ectopic gene expression}

To induce ectopic ci expression, flies containing the Gal4 ${ }^{C 591}$ driver were crossed to flies containing Nterminal fragments. These fragments encode a nuclear repressor form of $\mathrm{Ci}$ (Ci76) under UAS control. Ectopic $E(s p l)$ expression in the eye primordium was produced by crossing Gal4 ${ }^{C 591}$ flies to UAS-E(spl) flies. Ectopic expression of ptc was induced by crossing Gal4 ${ }^{C 591}$ flies to UASptc flies.

\section{Analysis of head morphology}

Flies heads were excised with a razor blade, washed in PBT (PBS plus 0.1\% Tween-20) and mounted in a 3:1 ethanol:glycerol solution. Wings were removed, washed briefly in PBT, and mounted onto a slide for further examination.

\section{In situ hybridization and immunocytochemistry}

$\mathrm{X}$-gal and antibody staining were performed as described by Royet and Finkelstein [1]. For in situ hybridization, larvae were grown in uncrowded conditions to obtain optimal imaginal disc morphology. Discs were dissected in PBS and fixed in 3\% formaldehyde in heptanesaturated PBS for 1 minute then in $6 \%$ formaldehyde for 20 minutes. They were then washed for $2 \times 5$ minutes in methanol, followed by $5 \times 5$ minutes in PBT. Discs were digested with $12 \mathrm{mg} / \mathrm{ml}$ proteinase $\mathrm{K}$ in PBS for 3 minutes and then incubated in $2 \mathrm{mg} / \mathrm{ml}$ glycine for 2 minutes. They were fixed again in $4 \%$ paraformaldehyde for 20 minutes and incubated with appropriate probes at $45^{\circ} \mathrm{C}$ overnight. Hybridization was carried out using digoxigenin-labeled DNA fragments following the protocol of Cubas et al [31]. To detect individual E(spl) bHLH, small fragments were used to minimize the possibility of cross-reactivity. Fragment used for $m 7$, SacI, was a $0.4-\mathrm{kb}$ fragment from cDNA clones [32] with vector-derived restriction site in brackets (Figure 2). The rest of the protocol was performed as described in [33]. Eye-antennal discs and adult heads were visualized and photographs were taken using a Zeiss Axioskop equipped with Nomarski optics.

\section{RESULTS}

The dorsal head capsule (also called the vertex) includes the region between the two compound eyes. The three ocelli lie on the triangular ocellar cuticle in the medial region of the vertex. Flanking the medial ocellus are two ocellar bristles, while two postvertical bristles lie near the lateral ocelli. A stereotypical pattern of smaller bristles (the intraocellar microchaetes) occupies the region within the three ocelli (Figure 3a).

In order to show the sensory origin of different components of the dorsal head capsule, L1 enhancer trap line is utilized where the lac $Z$ gene expression is restricted to the precursor cells of the ocelli and of the compound eye. During late third instar larval development (110-120

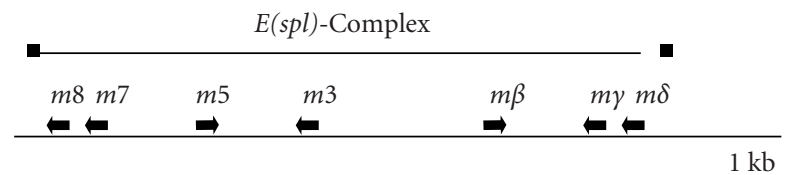

Figure 2. Organization of the $E(s p l)-C$ gene complex. Map of the $E(s p l)-C$. The line represents genomic DNA, distal is to the left, and the black arrows indicate transcripts encoding the $b H L H$ proteins $(\mathrm{m}, \mathrm{m} 5, \mathrm{~m} 7, \mathrm{~m} 8, \mathrm{~m} \beta, \mathrm{m} \gamma$, and $\mathrm{m} \delta)$. This diagram is modified from de Celis et al [20].

hours after egg laying) eye-antennal discs from L1 strain are stained for $\beta$-galactosidase activity, thus the photoreceptor cells of the compound eye and the precursors of the median and lateral ocelli are labeled (Figure 3b).

\section{hh is required for dorsal head formation}

Both $h h$ and $E(s p l)$ are expressed in the dorsal head primordium of the eye-antennal disc (Figures $3 c$ and $5 c$, respectively). $E(s p l)$ is also expressed in the antennal anlagen as well as in the morphogenetic furrow immediately adjacent to the region of $h h$ transcription. Eliminating $H h$ function during head development results in the deletion of the entire medial domain, including the interocellar cuticle and bristles, and the ocelli and their associated bristles (Figure 3d). This region is replaced by frons cuticle, which is normally confined to the mediolateral region of the head capsule.

\section{ptc and ci mediate the hh-dependent formation of the dorsal head}

Since $p t c$ is expressed in the ocellar primordium of wild-type eye discs (Figure 4a), the GAL4/UAS system [34] was used to overexpress UAS-ptc transgene across the entire dorsal head primordium (Figures $4 \mathrm{~b}, 4 \mathrm{c}$ ). Ectopic expression of ptc in Gal4 ${ }^{C 591} / \mathrm{UAS}$-ptc flies causes all three ocelli to become significantly smaller (Figure 4b).

The ci gene encodes a critical component of the $\mathrm{Hh}$ pathway. In order to determine if $h h$ mediates the dorsal head formation through $c i$, the two-component system (GAL4/UAS) was used to overexpress the repressor $\mathrm{N}$ terminal fragment of the $\mathrm{Ci}$ protein across the entire dorsal head primordium. Expression of this repressor fragment of $\mathrm{Ci}$ results in a head phenotype. This phenotype is almost identical to the $h h$ mutant phenotype (compare Figures $3 \mathrm{~d}$ and $4 \mathrm{~d}$ ).

\section{Loss of $N$ signaling deletes a specific region of the dorsal head}

Many studies have suggested a role of $\mathrm{N}$ during the dorsal head development. In order to confirm this role, the temperature-sensitive allele of $N, N^{t s 1}$, which fails to produce functional $\mathrm{N}$ protein at $29^{\circ} \mathrm{C}$, was used. Disrupting $\mathrm{N}$ function using a temperature-sensitive $\mathrm{N}$ allele eliminates the ocellar cuticle separating the three ocelli, resulting in the formation of a single giant ocellus (Figure $5 \mathrm{a}$ ). Ectopic expression of either $\mathrm{E}(\mathrm{spl}) \mathrm{m} 7$ or $\mathrm{E}(\mathrm{spl}) \mathrm{m} 8$ proteins was induced using the activator line 


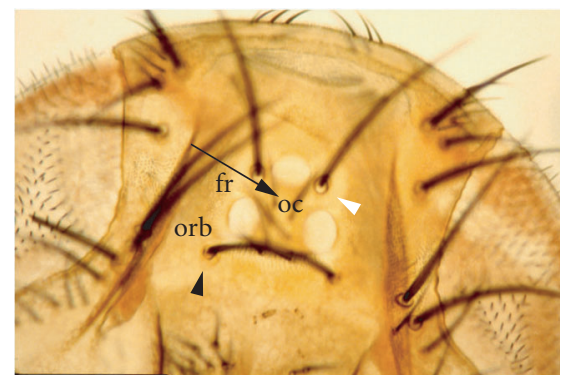

(a)

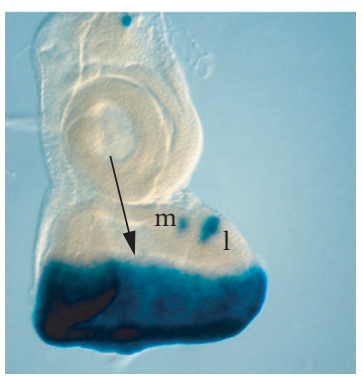

(b)

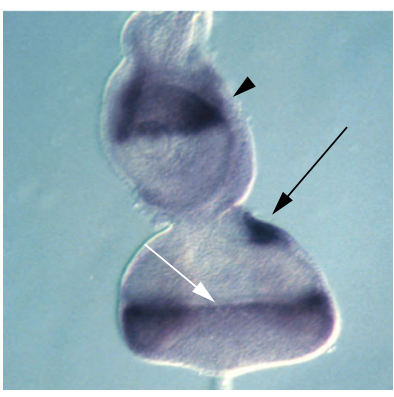

(c)

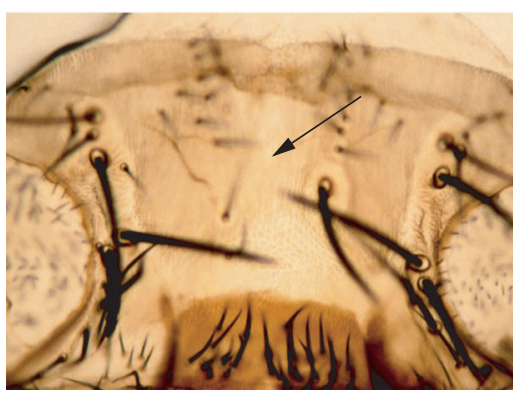

(d)

Figure 3. Hh is required for adult head development. (a) and (d) Dorsal views of the head capsules of flies of the indicated genotypes. (c) and (b) Eye-antennal discs. (a) Head of a wild-type fly with the interocellar cuticle (arrow). The normal appearance of the ocellar region (oc), frons (fr), and orbital region (orb) can be seen. The two ocellar bristles, which lie near the medial ocellus, are evident (white arrowhead indicates one of them). The two postvertical bristles (black arrowhead) lie near the lateral ocelli. The interocellar cuticle also contains 6-8 microchaetes (the interocellar bristles). (b) Third instar eye-antennal discs of L1 line were dissected and stained with $\mathrm{X}$-gal to detect $\beta$-galactosidase activity. Staining is present posterior to the morphogenetic furrow (arrow) and in the precursors of the median (m) and lateral (l) ocelli. (c) Wild-type eye-antennal disc hybridized with a digoxigenin-labeled $h h$ probe. In situ hybridization reveals that, in addition to being expressed in the dorsal head primordium (black arrow), $h h$ is expressed posterior to the morphogenetic furrow (white arrow). Parts of the antennal anlagen express $h h$ as well (arrowhead). (d) Head of an $h h^{t s 2} / h h^{t s 2} \mathrm{fly}$ raised at the restrictive temperature $\left(30^{\circ} \mathrm{C}\right)$ during the third larval instar. Loss of $h h$ function eliminates the entire medial domain of the dorsal head, including the ocelli, interocellar cuticle, and the ocellar, postvertical, and interocellar bristles. This region is replaced by ridged frons cuticle (arrow). Heads are at the same magnification. Discs are at the same magnification.

Gal4 ${ }^{C 591}$, which drives Gal4 expression across the dorsal head primordium of the eye-antennal imaginal disc (Figure 4c). Misexpression of either $m 8$ or $m 7$ caused a similar, but weaker phenotype, in which all ocellar bristles are eliminated and the ocelli are reduced in size (Figure 5b).

Figure $4 \mathrm{c}$ shows the expression pattern of the $m 7$ gene as detected by a cDNA probe that does not recognize the other mRNA transcripts in the $E(s p l)$ complex. In the third instar eye-antennal disc, $m 7$ is expressed in the ocellar region, the morphogenetic furrow and the antennal anlagen. The $m 8$ and $m \beta$ genes are also expressed in these cells (data is not shown).

\section{hh is epistatic to $E(s p l)$}

Eliminating $h h$ function during head development results in a marked reduction in the $E(s p l)$ expression during eye-antennal imaginal disc development. The E(spl) reduced expression is particularly clear at the ocellar primordium as well as at the morphogentic furrow (compare Figures $5 \mathrm{c}$ and $5 \mathrm{~d}$ ).

\section{DISCUSSION}

\section{hh signaling pathway mediates ocellar formation}

The involvement of the segment polarity gene $h h$ in patterning the ocellar region is supported by both genetic and molecular evidence. $h h$ is expressed in the ocellar region, overlapping with the expression domain of $o t d$. Loss of $h h$ activity eliminates the ocelli while ectopic $h h$ expression generates ectopic medial structures at more lateral positions. $h \mathrm{~h}$ is therefore necessary for the specification of the medial domain and is sufficient to direct more lateral regions of the dorsal head towards a medial fate. Further evidence indicated that $h h$ interacts genetically with $c i$ to form the ocellar region [35].

The ocellar phenotype associated with the loss of $h h$ function can be explained as follows. Hh inhibits the 


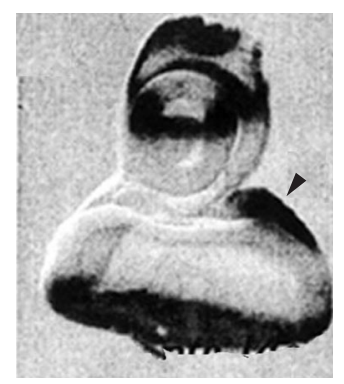

(a)

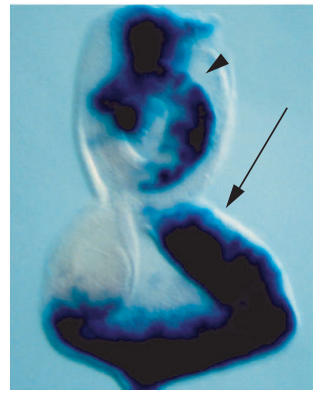

(c)

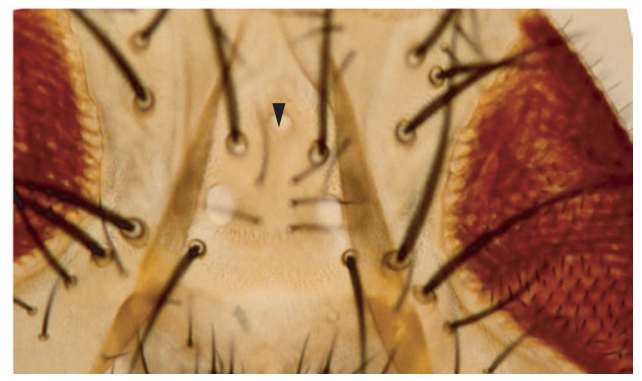

(b)

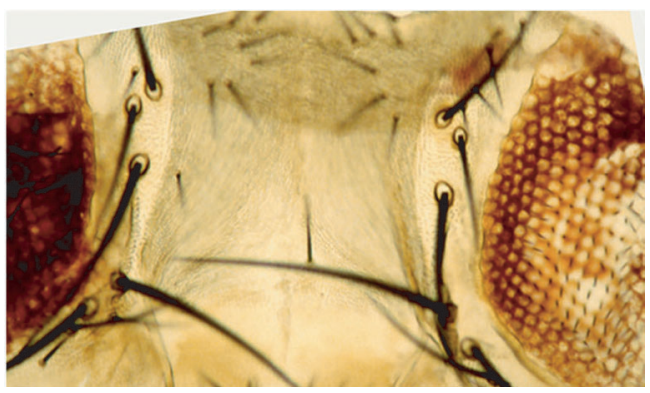

(d)

Figure 4. ptc and ci are crucial genes for ocellar development. (a) and (c) Eye-antennal discs stained with X-gal. (b) and (d) Dorsal views of the head capsules of flies of the indicated genotypes. (a) ptc-lacZ is expressed in the vertex primordium in the ptc-lacZ/+ eye discs (arrowhead). (b) Overexpression of ptc in the ocellar primordium in Gal4 ${ }^{C 591} /$ UAS-ptc flies results in smaller ocelli (arrowhead). (c) The Gal4 ${ }^{C 591}$ driver induces lacZ expression across the entire dorsal head primordium (arrow) as well as in the anlagen of the antenna (arrowhead) and in the compound eye. (d) In Gal4 ${ }^{C 591} /+$ and UAS-ci76/+ flies, the three ocelli and associated bristles are eliminated. The entire ocellar region is smaller.

production of Ci75 and causes an elevation of the levels of Ci155 protein in thoracic discs. Loss of $h h$ in the ocellar primordium might result in an upregulation of Ci75, therefore an ocelliless phenotype. Conversely, ectopic expression of $h$ in eye disc may cause a reduction of Ci75 level and an elevation of Ci155, either of which generates more ocelli. An exclusion of Ci75 from the ocellar primordium is also required for the normal ocellar formation as ectopic expression of Ci75 in the eye-antennal disc eliminates the ocelli (Figure 4d). Similarly, in wing disc, Hh has been shown to specify the A-cell affinity through a transcriptional response mediated by $\mathrm{Ci}[36,37]$. Segregation of $\mathrm{A}$ and $\mathrm{P}$ cells was shown to critically depend on the Ci155/Ci75 ratio found in several rows of A-cells next to the compartment boundary. In fact, A cells containing Ci75 intermingle better with $\mathrm{P}$ cells than cells that lack $\mathrm{Ci}$ suggesting that $\mathrm{Ci} 75$ modifies the A-cell affinity to resemble the P-cell affinity [38].

Although both Ci75 and Ci155 contain the zinc finger domain, previous analysis of their activities showed that they differently behave. Ci75 lacks a cytoplasmic tethering domain and is therefore located in the nucleus while Ci155 protein is mainly detected in the cytoplasm. Ci75 represses the expression of $h h, p t c$, and $w g$. Ci155 acts as a transactivator in embryos and turns on the expression of ptc and epidermal growth factor receptor (EGFR) in imaginal discs.

This report provides evidence that Ci75 is capable of producing a phenotype that is opposite to that produced by Ci155 in the same tissue. The Ci155 translation product forms a complex with the kinesin-related Costal2 and the Ser-Thr kinase Fused. This complex is retained in the cytosol through its tight binding to microtubules. In the absence of Hh signaling, release of $\mathrm{Ci}$ from this complex involves proteolytic processing to $\mathrm{Ci} 75$, which is translocated into the nucleus and behaves as a repressor. In Hhreceiving cells, signaling releases Ci155 from the complex, prevents its proteolytic processing, stimulates its maturation into a labile transcriptional activator, and promotes its nuclear import [39].

\section{N pathway is required for dorsal head development}

During Drosophila embryogenesis, $N$ was the first gene shown to be necessary for partitioning of cell fates within the neurogenic region. Deletion of specific components of the $\mathrm{N}$ signaling pathway leads to neural hypertrophy. In addition, $\mathrm{N}$ is required for the specification of embryonic mesoderm. Absence of $\mathrm{N}$ during postembryonic 


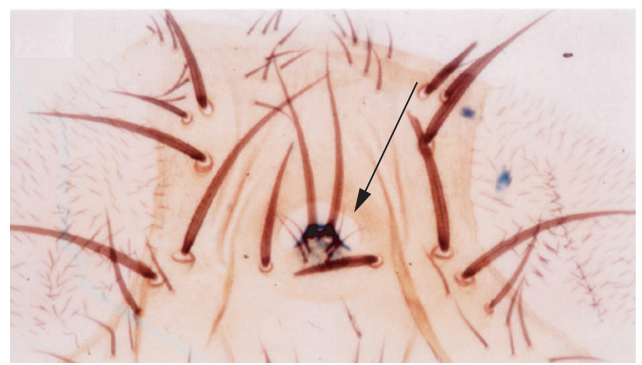

(a)

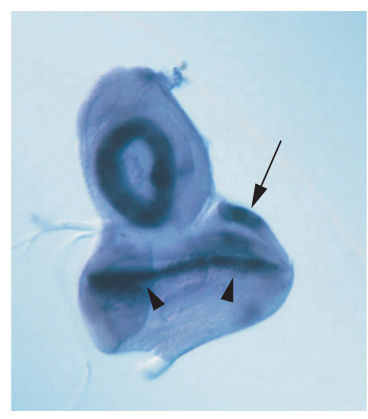

(c)

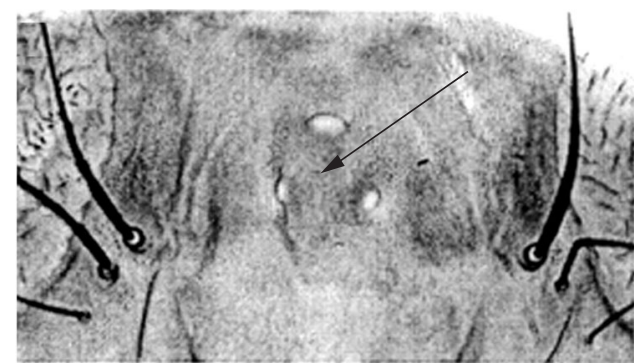

(b)

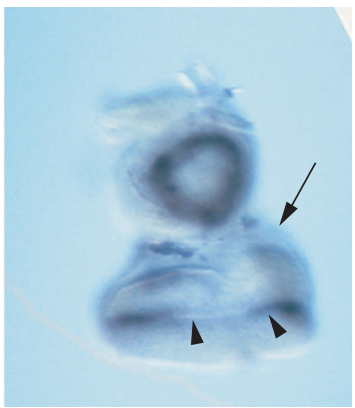

(d)

Figure 5. Regulation of $E(\mathrm{spl})$ expression by $h h$. (a) and (b) Dorsal views of the head capsules of flies of the indicated genotypes. (c) and (d) Eye-antennal discs hybridized with a digoxigenin-labeled $E(s p l)$ probe. (a) $N^{t s 1} / Y$ fly head. The ocelli are fused (arrow) and the intraocellar cuticle is lost. (b) A complete loss of bristles (arrow) was obtained in Gal4 ${ }^{C 591} /+$ and $U A S-m 7 /+$ flies. (c) In situ hybridization screening using $m 7 \mathrm{cDNA}$ demonstrates the presence of $m 7 \mathrm{mRNA}$ in ocellar primordium (arrow), morphogenetic furrow (arrowheads), and antennal disc. (d) Elimination of $h h$ in $h h^{t s 1} / h h^{t s 1}$ eye imaginal discs abolishes $E(s p l)$ expression in the ocellar primordium (arrow) and significantly disrupts its expression in the morphogenetic furrow (arrowheads).

development has revealed multiple roles for $\mathrm{N}$ and its partners in the specification of various cell types. Adult viable mutations in $N$ and other components of this signaling pathway affect the development of bristles, eyes, wings, veins, and legs. Studies of the expression of $N$ and other components of the $\mathrm{N}$ signaling pathway are correlated well with their above roles during embryogenesis and larval development [40].

The function of the $\mathrm{N}$ signaling pathway in the compound eye portion of the eye disc and wing disc has been very well elucidated [41]. However, the role of $\mathrm{N}$ in the ocellar region has not been very well characterized. Lossof-function experiment using the temperature-sensitive $N$ allele $\left(N^{t s 1}\right)$ eliminates the ocellar cuticle separating the three ocelli, resulting in the formation of a single giant ocellus (Figure 5a). The intraocellar microchaetes, as well as one or both of the ocellar bristles, are eliminated in these flies. More lateral head structures, such as the postvertical bristles, are not affected. Consistent with previous reports, these flies also exhibit a loss of the wing margin, a rough eye phenotype, as well as supernumerary bristles.

The reduction of $\mathrm{N}$ function during larval development generally leads to changes in cell fate specification.
This study, however, suggests that the loss of $N$ in the ocellar region results in the loss of a population of cells instead of a cell fate change. Previous analysis of $\mathrm{N}$ in Drosophila and its related proteins in vertebrates indicated that the $\mathrm{N}$ signaling cascade may participate in the process of proliferation. There are at least three $N$-related genes in the mouse. Loss-of-function in one (Notch 1) of these alleles leads to widespread cell death in central and peripheral nervous systems [42].

This report demonstrates that $(E(s p l))$, a downstream component of the $\mathrm{N}$ signaling pathway, is also expressed in the head vertex. Overexpression of this gene not only deletes all the ocelli-associated bristles but it also forms a smaller ocelli (Figure 5b). This functional role of $\mathrm{E}(\mathrm{spl})$ is consistent with its distinct pattern of expression in the ocellar primordium of the eye disc. At least three $(m 7, m 8$, and $m \beta$ ) of the seven $E(s p l) b H L H$ genes are expressed in the precursor cells of the ocelli. Comparison of $m 7$ expression with that of en shows that $m 7$ is expressed in the epidermal precursor cells that separate the ocelli. This expression pattern of $E(s p l)$ is strongly disrupted by the elimination of $h h$ function in this region (Figures $5 c$ and 5d). Cross-talk between the $\mathrm{Hh}$ and $\mathrm{N}$ signaling pathways has been shown to be crucial for assigning overlapping 
A/P positions to the L3 vein and associated sensory organs [43]. $\mathrm{N}$ and Hh signaling have also been shown to exist in a delicate balance to allow bristle and sensory organ differentiation along the adult wing margin [44]. Results presented here provide a new evidence that $\mathrm{N}$ signaling pathway is a new downstream target to the long list of genes that $h$ h regulates during development [45]. Similarly, shh has been shown to act upstream of the N Pathway during arterial endothelial differentiation [46].

\section{ACKNOWLEDGMENT}

I am indebted to Dr. Robert Finkelstein at NIH for all his assistance throughout the course of this work.

\section{REFERENCES}

[1] Royet J, Finkelstein R. Pattern formation in Drosophila head development: the role of the orthodenticle homeobox gene. Development. 1995; 121(11):3561-3572.

[2] Oh CT, Kwon SH, Jeon KJ, Han P-L, Kim SH, Jeon S-H. Local inhibition of Drosophila homeoboxcontaining neural dorsoventral patterning genes by Dpp. FEBS Lett. 2002;531(3):427-431.

[3] Zhang XM, Ramalho-Santos M, McMahon AP. Smoothened mutants reveal redundant roles for Shh and Ihh signaling including regulation of $\mathrm{L} / \mathrm{R}$ asymmetry by the mouse node. Cell. 2001;105(6):781792. [published correction appears in Cell. 2001; 106(2):253].

[4] Hammerschmidt M, Brook A, McMahon AP. The world according to hedgehog. Trends Genet. 1997; 13(1):14-21.

[5] Alcedo J, Zou Y, Noll M. Posttranscriptional regulation of smoothened is part of a self-correcting mechanism in the Hedgehog signaling system. Mol Cell. 2000;6(2):457-465.

[6] Ingham PW, Nystedt S, Nakano Y, et al. Patched represses the Hedgehog signaling pathway by promoting modification of the Smoothened protein. Curr Biol. 2000;10(20):1315-1318.

[7] Ramirez-Weber FA, Casso DJ, Aza-Blanc P, Tabata T, Kornberg TB. Hedgehog signal transduction in the posterior compartment of the Drosophila wing imaginal disc. Mol Cell. 2000;6(2):479-485.

[8] Strutt H, Thomas C, Nakano Y, et al. Mutations in the sterol-sensing domain of Patched suggest a role for vesicular trafficking in Smoothened regulation. Curr Biol. 2001;11(8):608-613. [published correction appears in Curr Biol. 2001; 11(14):1153].

[9] Nybakken K, Perrimon NP. Hedgehog signal transduction: recent findings. Curr Opin Genet Dev. 2002;12(5):503-511.

[10] Schwartz C, Locke J, Nishida C, Kornberg TB. Analysis of cubitus interruptus regulation in Drosophila embryos and imaginal disks. Development. 1995; 121(6):1625-1635.
[11] Aza-Blanc P, Ramirez-Weber FA, Laget MP, Schwartz C, Kornberg TB. Proteolysis that is inhibited by hedgehog targets Cubitus interruptus protein to the nucleus and converts it to a repressor. Cell. 1997; 89(7):1043-1053.

[12] Nybakken K, Perrimon N. Hedgehog signal transduction: recent findings. Curr Opin Genet Dev. 2002;12(5):503-511.

[13] Alexandre C, Jacinto A, Ingham PW. Transcriptional activation of hedgehog target genes in Drosophila is mediated directly by the cubitus interruptus protein, a member of the GLI family of zinc finger DNAbinding proteins. Genes Dev. 1996;10(16):20032013.

[14] Marigo V, Davey RA, Zuo Y, Cunningham JM, Tabin CJ. Biochemical evidence that patched is the Hedgehog receptor. Nature. 1996;384(6605):176-179.

[15] Royet J, Finkelstein R. Hedgehog, wingless and orthodenticle specify adult head development in Drosophila. Development. 1996;122(6):1849-1858.

[16] Slusarski DC, Motzny CK, Holmgren R. Mutations that alter the timing and pattern of cubitus interruptus gene expression in Drosophila melanogaster. Genetics. 1995;139(1):229-240.

[17] Royet J, Finkelstein R. Establishing primordia in the Drosophila eye-antennal imaginal disc: the roles of decapentaplegic, wingless and hedgehog. Development. 1997;124(23):4793-4800.

[18] Redmond L, Ghosh A. The role of Notch and Rho GTPase signaling in the control of dendritic development. Curr Opin Neurobiol. 2001;11(1):111-117.

[19] Artavanis-Tsakonas S, Matsuno K, Fortini ME. Notch signaling. Science. 1995;268(5208):225-232.

[20] de Celis JF, de Celis J, Ligoxygakis P, Preiss A, Delidakis C, Bray S. Functional relationships between Notch, $S u(H)$ and the bHLH genes of the E(spl) complex: the $E(s p l)$ genes mediate only a subset of Notch activities during imaginal development. Development. 1996;122(9):2719-2728.

[21] Xu T, Caron LA, Fehon RG, Artavanis-Tsakonas S. The involvement of the Notch locus in Drosophila oogenesis. Development. 1992;115(4):913-922.

[22] Bate M, Rushton E, Frasch M. A dual requirement for neurogenic genes in Drosophila myogenesis. Dev Suppl. 1993:149-161.

[23] Lundkvist J, Lendahl U. Notch and the birth of glial cells. Trends Neurosci. 2001;24(9):492-494.

[24] Selkoe D. Notch signaling in T cell development. Curr Opin Immunol. 2000;12:166-172.

[25] Fortini ME, Artavanis-Tsakonas S. The suppressor of hairless protein participates in Notch receptor signaling. Cell. 1994;79(2):273-282.

[26] Chandra S, Ahmed A, Vaessin H. The Drosophila IgC2 domain protein Friend-of-Echinoid, a paralogue of Echinoid, limits the number of sensory organ precursors in the wing disc and interacts with the Notch signaling pathway. Dev Biol. 2003;256(2): 302-316. 
[27] Ma C, Zhou Y, Beachy PA, Moses K. The segment polarity gene hedgehog is required for progression of the morphogenetic furrow in the developing Drosophila eye. Cell. 1993;75(5):927-938.

[28] Rulifson EJ, Blair SS. Notch regulates wingless expression and is not required for reception of the paracrine wingless signal during wing margin neurogenesis in Drosophila. Development. 1995;121(9): 2813-2824.

[29] Mozer BA, Benzer S. Ingrowth by photoreceptor axons induces transcription of a retrotransposon in the developing Drosophila brain. Development. 1994; 120(5):1049-1058.

[30] Amin A, Finkelstein R. Epidermal growth factor receptor signaling activates orthodenticle expression during Drosophila head development. DNA Cell Biol. 2000;19(11):631-638.

[31] Cubas P, de Celis JF, Campuzano S, Modolell J. Proneural clusters of achaete-scute expression and the generation of sensory organs in the Drosophila imaginal wing disc. Genes Dev. 1991;5(6):996-1008.

[32] Delidakis C, Artavanis-Tsakonas S. The Enhancer of split [E(spl)] locus of Drosophila encodes seven independent helix-loop-helix proteins. Proc Natl Acad Sci USA. 1992;89(18):8731-8735.

[33] Finkelstein R, Perrimon N. The orthodenticle gene is regulated by bicoid and torso and specifies Drosophila head development. Nature. 1990;346(6283):485488.

[34] Brand AH, Perrimon N. Targeted gene expression as a means of altering cell fates and generating dominant phenotypes. Development. 1993;118:401-415.

[35] Amin A, Li Y, Finkelstein R. Hedgehog activates the EGF receptor pathway during Drosophila head development. Development. 1999;126(12):2623-2630.

[36] Dahmann C, Basler K. Opposing transcriptional outputs of Hedgehog signaling and engrailed control compartmental cell sorting at the Drosophila A/P boundary. Cell. 2000;100(4):411-422.

[37] Ingham PW, McMahon AP. Hedgehog signaling in animal development: paradigms and principles. Genes Dev. 2001;15(23):3059-3087.

[38] Tepass U, Godt D, Winklbauer R. Cell sorting in animal development: signaling and adhesive mechanisms in the formation of tissue boundaries. Curr Opin Genet Dev. 2002;12(5):572-582.

[39] Arst HN Jr, Peñalva MA. pH regulation in Aspergillus and parallels with higher eukaryotic regulatory systems. Trends Genet. 2003;19(4):224-231.

[40] de Celis JF, Bray S, Garcia-Bellido A. Notch signaling regulates veinlet expression and establishes boundaries between veins and interveins in the Drosophila wing. Development. 1997;124(10):1919-1928.

[41] Kumar JP, Moses K. EGF receptor and Notch signaling act upstream of Eyeless/Pax6 to control eye specification. Cell. 2001;104(5):687-697.

[42] Swiatek PJ, Lindsell CE, del Amo FF, Weinmaster G, and Gridley T. Notch 1 is essential for postimplanta- tion development in mice. Genes Dev. 1994;8:707719.

[43] Crozatier M, Glise B, Khemici V, Vincent A. Veinpositioning in the Drosophila wing in response to Hh; new roles of Notch signaling. Mech Dev. 2003; 120(5):529-535.

[44] Glise B, Jones DL, Ingham PW. Notch and Wingless modulate the response of cells to Hedgehog signaling in the Drosophila wing. Dev Biol. 2002;248(1):93106.

[45] McMahon AP. More surprises in the Hedgehog signaling pathway. Cell. 2000;100(2):185-188.

[46] Lawson ND, Vogel AM, Weinstein BM. Sonic hedgehog and vascular endothelial growth factor act upstream of the Notch pathway during arterial endothelial differentiation. Dev Cell. 2002;3(1):127136.

\footnotetext{
* E-mail: a.amin@uaeu.ac.ae Fax: +9713 7671291; Tel: +97137064389
} 

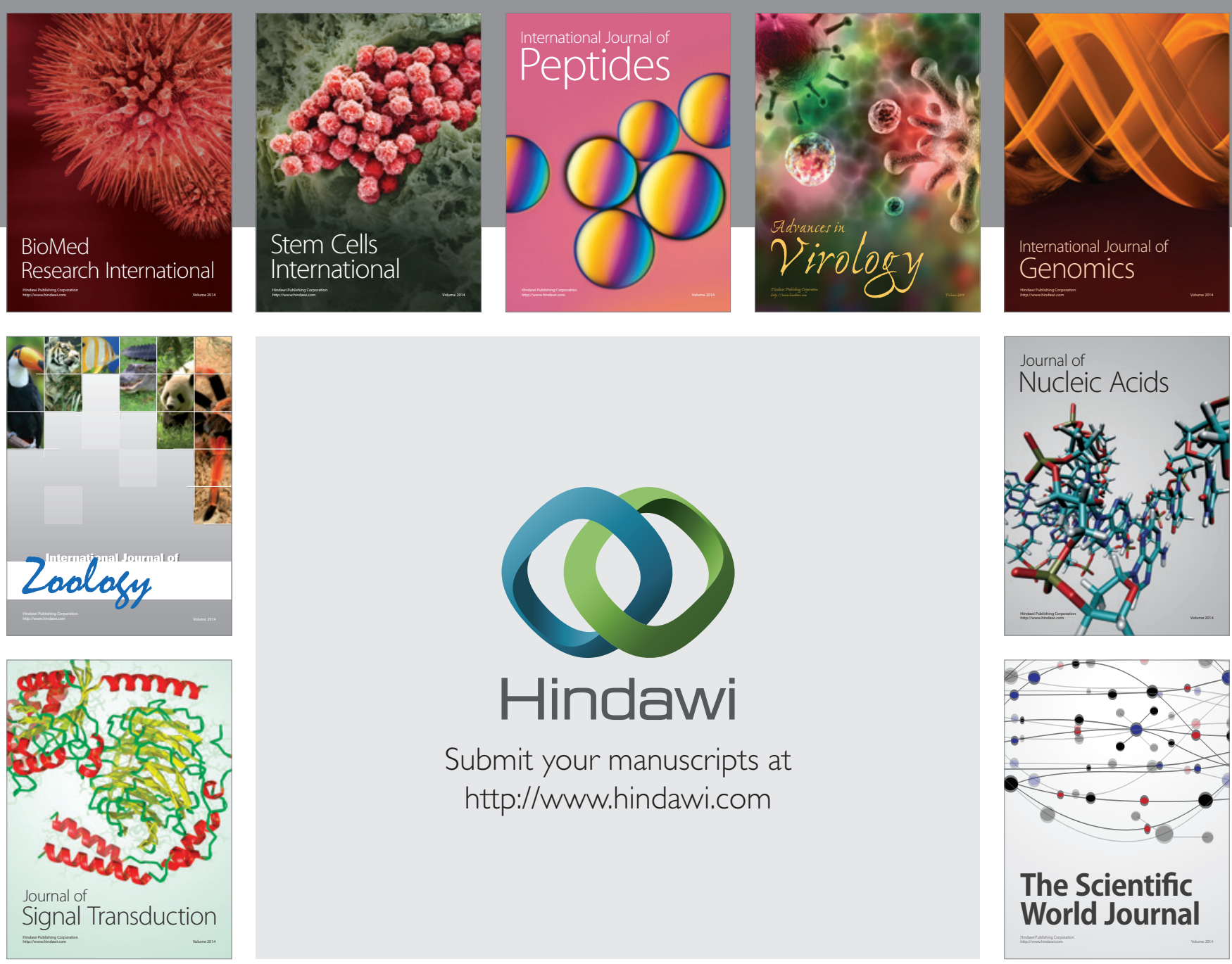

Submit your manuscripts at

http://www.hindawi.com
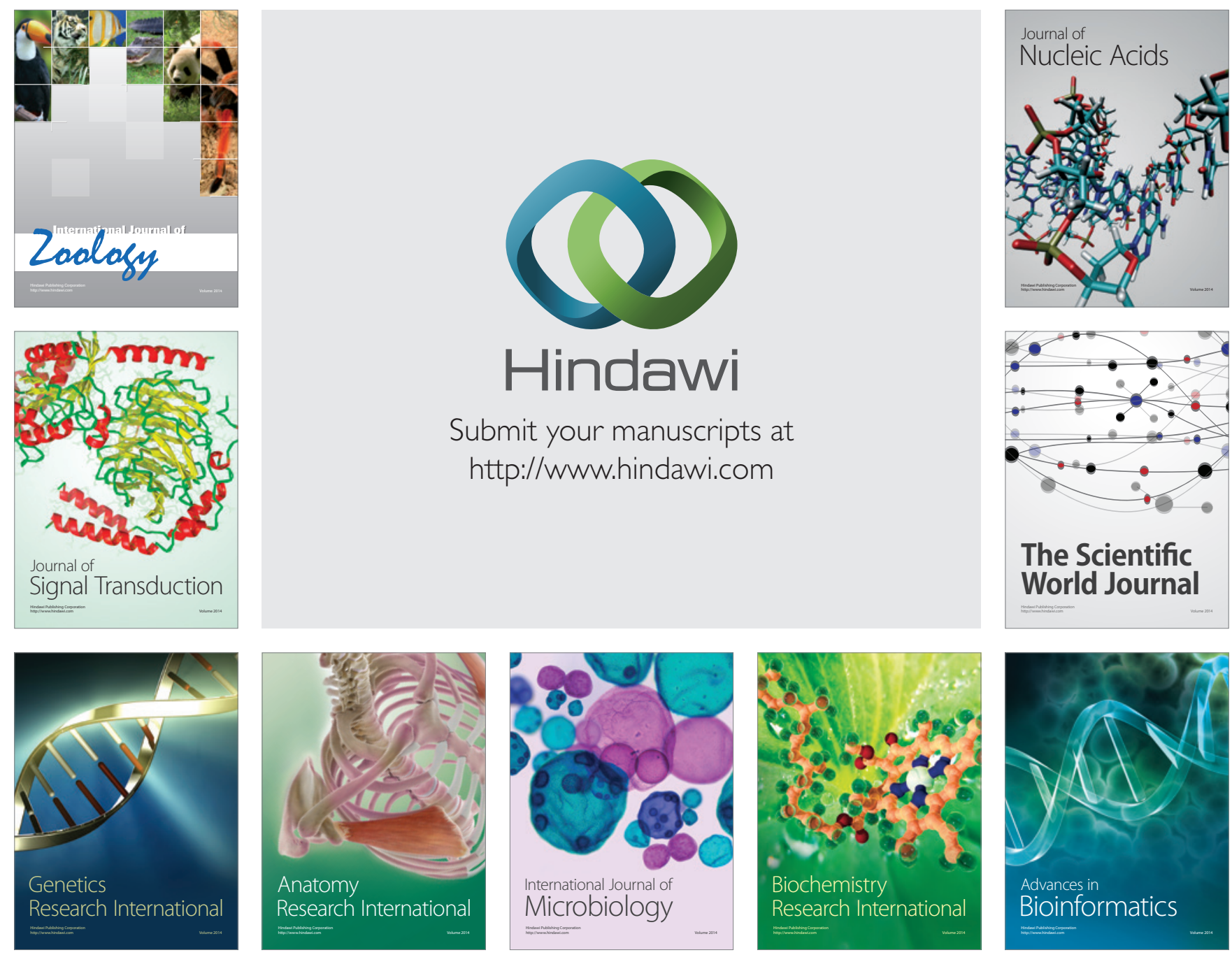

The Scientific World Journal
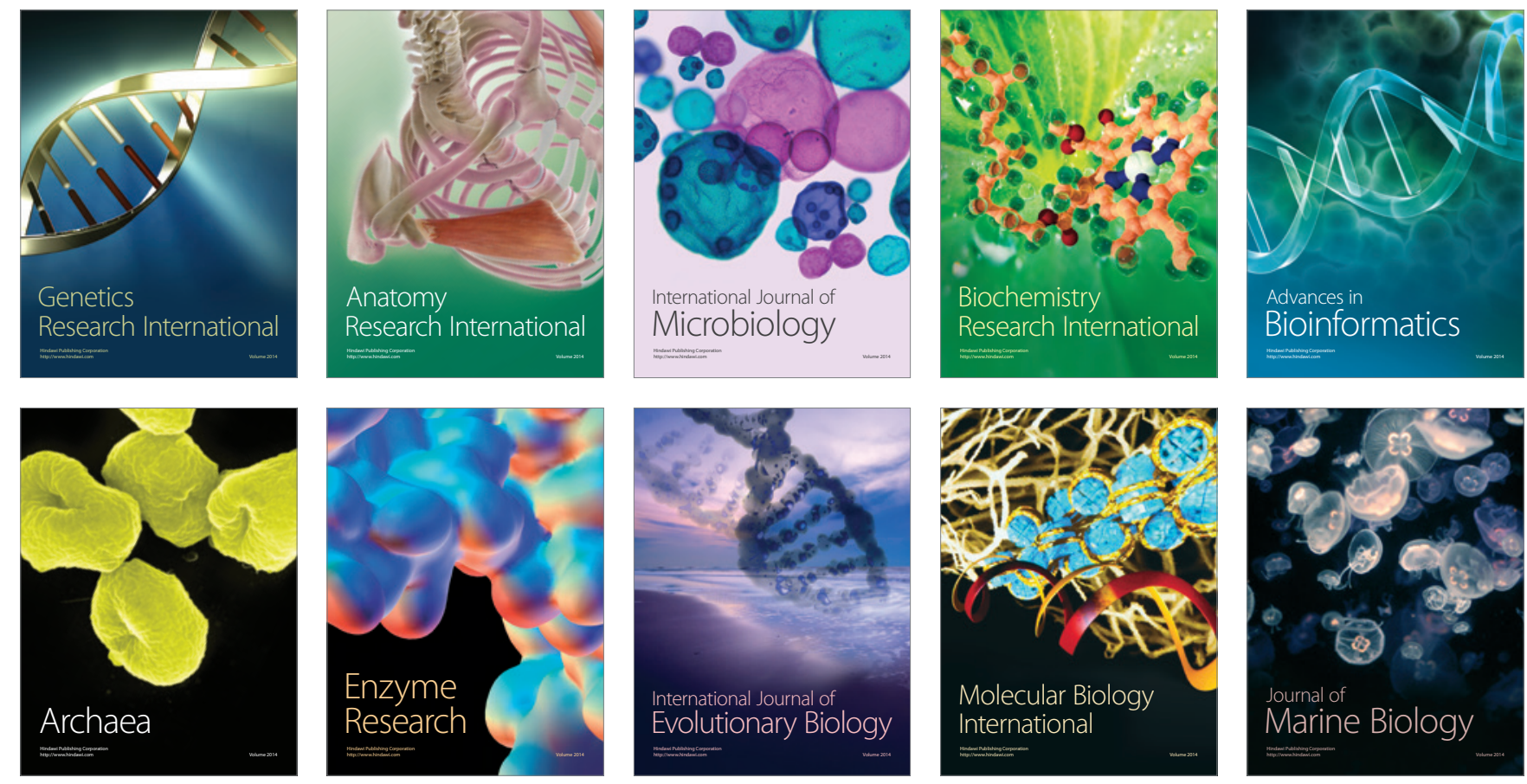